\title{
REFLECTION OF WATER WAVES BY A NEARLY VERTICAL POROUS WALL
}

\author{
A. CHAKRABARTI and T. SAHOO 1
}

(Received 10 September 1993; revised 4 July 1994)

\begin{abstract}
The problem of reflection of water waves by a nearly vertical porous wall has been investigated. A perturbational analysis has been applied for the first order correction to be employed to the corresponding vertical wall problem. The Green's function technique has been used to obtain the solution of the boundary value problem at hand, after utilising a mixed Fourier transform together with an idea involving the regularity of the transformed function along the real axis. The cases of fluid of finite as well as infinite depth have been taken into consideration. Particular shapes of the wall have been considered and numerical results are also discussed.
\end{abstract}

\section{Introduction}

Boundary value problems associated with the two-dimensional Laplace's equation involving a quarter plane occur in a natural way in the study of propagation of surface water waves concerning fluid of infinite depth having a vertical wall at one end. A similar situation arises even in the case of fluid occupying a solid bottom at a finite depth. Several methods of attack have been demonstrated (see Mandal and Kar [6], Chakrabarti [1], Chwang [2], Shaw [7], Mandal and Chakrabarti [5]) to handle such boundary problems in the literature. Recently Mandal and Kar [6] have devised a perturbational approach, similar to the one utilised in the problems treated in Shaw [7] and Mandal and Chakrabarti [5] to handle a boundary value problem where the study of reflection of surface water waves by a nearly vertical barrier (see Mandal and Kar [6], Shaw [7], Mandal and Chakrabarti [5]) is of prime concern. In the present paper, we have further generalised the approach of Mandal and Kar [6] to analyse the effect of porosity on the nearly vertical wall. We have also considered both the problems of infinite as well as finite depths of fluid. By using the perturbational approach of the previous workers, we have broken both the problems under consideration into two

\footnotetext{
'Department of Mathematics, Indian Institute of Science, Bangalore 560012 , India.

(C) Australian Mathematical Society, 1996, Serial-fee code 0334-2700/95
} 


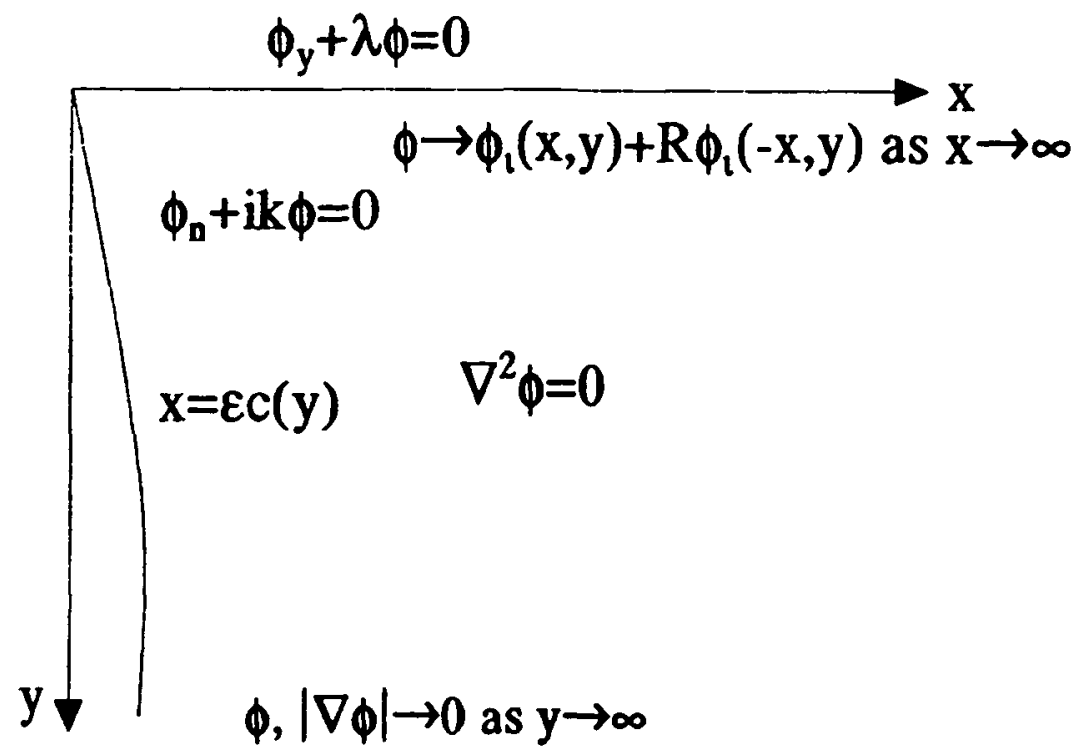

FIGURE 1. Water of infinite depth (Problem $P_{1}$ ).

boundary value problems for Laplace's equation, and have solved these problems by utilising a mixed Fourier-type integral transform (see Sneddon [8]) along with the use of certain regularity conditions to be satisfied by the transformed functions. The final results for the reflection coefficient have been presented in closed analytic forms, and it is observed that the corresponding results of Mandal and Kar [6] follow as a particular limiting case of the general problems considered here when the porosity is absent. Similar surface wave problems have been considered earlier by Kachoyan and McKee [4].

Certain numerical results involving the first order correction $R_{1}$ to the reflection coefficient have been calculated, and such results have been presented graphically in the case of water of finite as well as infinite depths.

\section{Formulation of the problem}

The problems under study are two-dimensional in nature. We employ a rectangular Cartesian co-ordinate system $(x, y)$. The boundary $x=\epsilon c(y)$ with $c(0)=0, y>0$ for water of infinite depth $(0<y<h$, for water of finite depth), represents the porous nearly vertical wall, $c(y)$ being a continuous bounded function of $y$ with the $y$-axis being taken in the vertically downward direction and $\epsilon$ being a small parameter. See Figures 1 and 2 for the geometrical description of the two problems under consideration. It is assumed that the fluid is incompressible, the motion is irrotational and 


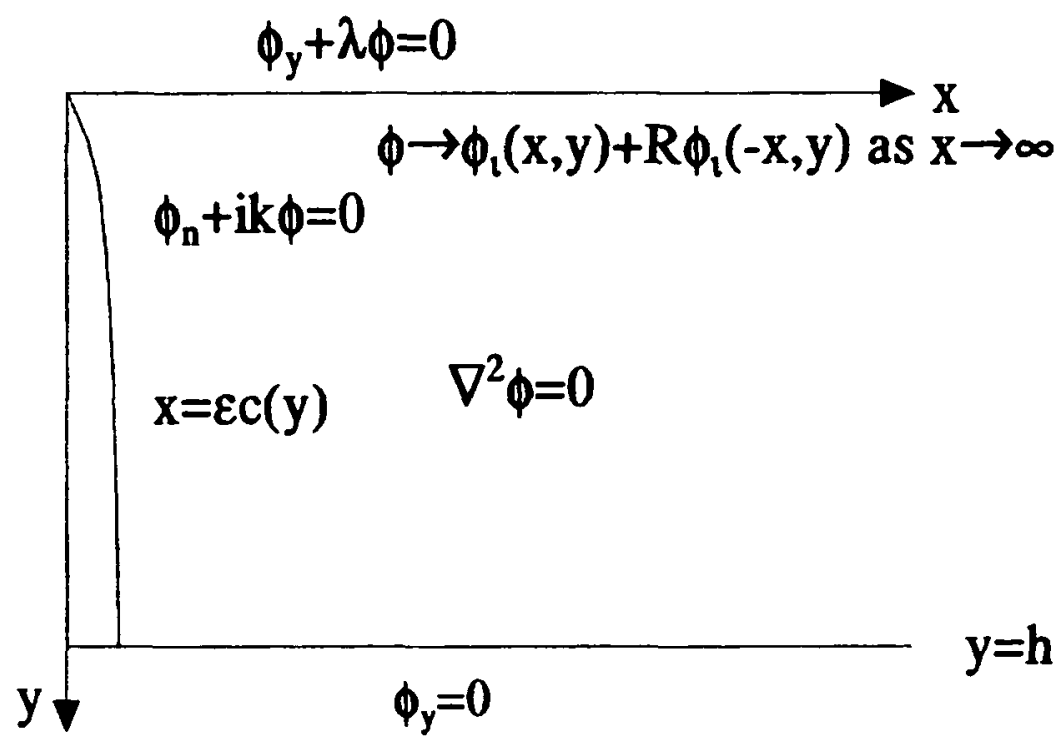

FIGURE 2, Water of finite depth (Problem $P_{2}$ ).

the total potential simple harmonic in time. This ensures the existence of a velocity potential $\Phi(x, y, t)=\operatorname{Re}\left(\phi(x, y) e^{-i \omega t}\right)$, "Re" denoting the "real part". We consider here the problem of the reflection of water waves having the incident potential $\Phi_{i}(x, y, t)=\operatorname{Re}\left(\phi_{i}(x, y) e^{-i \omega t}\right)=\operatorname{Re}\left(e^{(-\lambda y-i \lambda x-i \omega t)}\right)$ in the case of water of infinite $\operatorname{depth}\left(\right.$ and $\Phi_{i}(x, y, t)=\operatorname{Re}\left(\phi_{i}(x, y) e^{-i \omega t}\right)=\operatorname{Re}\left(\left(\cosh k_{0}(h-y) / \cosh k_{0} h\right) e^{-i k_{0} x-i \omega t}\right)$ in the case of water of finite depth, where $k_{0}$ is the positive real root of the equation $\lambda=m \tanh (m h))$ with $\lambda=\omega^{2} / g, \omega$ being the angular frequency and $g$ the acceleration due to gravity. We will drop the factor $e^{-i \omega t}$ throughout the rest of this paper. The effect of the nearly vertical barrier will be studied completely up to the order of $\epsilon$ in both the cases of finite and infinite depth of water by way of determining the velocity potential $\phi(x, y)$, where $\phi$ satisfies the PDE

$$
\nabla^{2} \phi=0 \text { in the fluid region, }
$$

with

$$
\begin{array}{cc}
\frac{\partial \phi}{\partial y}+\lambda \phi=0 \quad \text { on } & y=0, x-\epsilon c(y)>0, \\
\frac{\partial \phi}{\partial n}+i k \phi=0 \quad \text { on } & x-\epsilon c(y)=0, y>0 .
\end{array}
$$

( $\frac{\partial}{\partial n}$ denotes the derivative in the inward normal direction to the boundary $x=\epsilon c(y)$ ) (An explanation of the derivation of this boundary condition follows.)

$\phi,|\nabla \phi| \rightarrow 0$ as $y \rightarrow 0$ in the case of water of infinite depth, 


$$
\frac{\partial \phi}{\partial y}=0 \quad \text { on } \quad y=h \quad \text { in the case of water of finite depth, }
$$

and

$$
\phi \rightarrow \phi_{i}(x, y)+R \phi_{i}(-x, y) \quad \text { as } \quad x \rightarrow \infty
$$

in both cases considered, where $R$ is an unknown constant representing the "reflection coefficient", to be determined.

In order to arrive at the boundary condition (3) along the porous wall, we observe, from Bernoulli's equation, that

$$
P(x, y, t)=-\rho \Phi_{t} \quad \text { for small motion, }
$$

where $P(x, y, t)$ is the hydrodynamic pressure on the wall $x=\epsilon c(y)$. Assuming the wall to be made of fine pores we have that

$$
\frac{\partial \Phi}{\partial n}=-\frac{b}{\mu} P(x, y, t) \quad \text { on } \quad x=\epsilon c(y) .
$$

The normal velocity of the fluid passing through the porous wall is proportional to the pressure exerted on the wall, and $\mu$ is the dynamic coefficient of viscosity and $b$ is a constant which has the dimension of length (see Chwang [2]). On the other side of the wall the pressure is constant. Without loss of generality we can take the pressure to be zero there. Combining (7) and (8) we have that

$$
\frac{\partial \Phi}{\partial n}=\frac{b \rho}{\mu} \Phi_{t} \quad \text { on } \quad x=\epsilon c(y)
$$

Again, since $\Phi(x, y, t)=\operatorname{Re}\left(\phi(x, y) e^{-i \omega t}\right)$, we can express the above condition in the form

$$
\frac{\partial \phi}{\partial n}=-\frac{i b \rho \omega}{\mu} \phi=-i k \phi \quad \text { on } \quad x=\epsilon c(y),
$$

where $k=\frac{b \rho \omega}{\mu}$. This explains the occurrence of the condition (3) to be used. Condition (3) can be further expressed in the approximate form, for small values of $\epsilon$, after using a Taylor-expansion procedure (see Mandal and Kar [6], Shaw [7]) and we obtain that

$$
\frac{\partial \phi}{\partial x}-\epsilon \frac{d}{d y}\left(c(y) \frac{\partial \phi}{\partial y}\right)=-i k\left[\phi+\epsilon c(y) \frac{\partial \phi}{\partial x}\right] \quad \text { on } \quad x=0 .
$$

We shall employ a perturbation expansion of the potential $\phi$ and the reflection coefficients $R$ (as in the work of Mandal and Chakrabarti [5], Mandal and Kar [6]) in the forms

$$
\begin{aligned}
\phi(x, y, \epsilon) & =\phi_{0}(x, y)+\epsilon \phi_{1}(x, y)+o\left(\epsilon^{2}\right), \\
R & =R_{0}+\epsilon R_{1}+o\left(\epsilon^{2}\right) .
\end{aligned}
$$


Then the problems under consideration reduce to two boundary value problems for $\phi_{0}$ and $\phi_{1}$ each as explained below. Using the notation $P_{1}$ for the problem which corresponds to the case of water of infinite depth and $P_{2}$ for the problem which corresponds to the case of fluid of finite depth, and writing $L_{1}=(y: 0<y<\infty)$, $L_{2}=(y: 0<y<h)$, the mathematical forms of the various boundary value problems to be solved are given as follows.

\subsection{Problem for $\phi_{0}(x, y)$}

$$
\begin{array}{r}
\nabla^{2} \phi_{0}=0 \text { in the region } x>0, \\
\frac{\partial \phi_{0}}{\partial x}+i k \phi_{0}=0 \text { on } x=0, y \in L_{1} \text { for } P_{1}\left(y \in L_{2} \text { for } P_{2}\right), \\
\frac{\partial \phi_{0}}{\partial y}+\lambda \phi_{0}=0 \text { on } y=0, x>0 \text { for both } P_{1} \text { and } P_{2} \\
\left|\nabla \phi_{0}\right| \rightarrow 0 \text { as } y \rightarrow \infty \text { for } P_{1}, \\
\frac{\partial \phi_{0}}{\partial y}=0 \text { on } y=h \text { for } P_{2}, \\
\phi_{0} \rightarrow \phi_{i}(x, y)+R_{0} \phi_{i}(-x, y) \text { as } x \rightarrow \infty \text { in both cases. }
\end{array}
$$

\subsection{Problem for $\phi_{1}(x, y)$}

$$
\nabla^{2} \phi_{1}=0 \text { in the region } x>0,
$$

$$
\frac{\partial \phi_{1}}{\partial x}+i k \phi_{1}=f(y) \text { on } x=0, y \in L_{1} \text { for } P_{1}\left(y \in L_{2} \text { for } P_{2}\right),
$$

where $f(y)=\frac{d}{d y}\left[c(y) \frac{\partial \phi_{0}(0, y)}{\partial y}\right]-i k c(y) \frac{\partial \phi_{0}(0, y)}{\partial x}$,

$$
\begin{gathered}
\frac{\partial \phi_{1}}{\partial y}+\lambda \phi_{1}=0 \quad \text { on } y=0, x>0 \text { for both } P_{1} \text { and } P_{2}, \\
\left|\nabla \phi_{1}\right| \rightarrow 0 \quad \text { as } y \rightarrow \infty \text { for } P_{1}, \\
\frac{\partial \phi_{1}}{\partial y}=0 \quad \text { on } y=h \text { for } P_{2},
\end{gathered}
$$

and

$$
\phi_{1} \rightarrow R_{1} \phi_{i}(-x, y) \text { as } x \rightarrow \infty \text { in both cases. }
$$

In the next section, we present the methods of solution of the above boundary value problems. 


\section{The methods of solution}

3.1. Determination of $\phi_{0}(x, y)$ (Infinite depth case) We put

$$
\phi_{0}(x, y)=\psi_{0}(x, y)+\phi_{i}(x, y)+R_{0} \psi_{i}(-x, y)
$$

for the problem $P_{1}$ and use an integral transform of $\psi_{0}(x, y)$ given by

$$
\hat{\psi}_{0}(\xi, y)=\int_{0}^{\infty} \psi_{0}(x, y)(\xi \cos \xi x-i k \sin \xi x) d x .
$$

Substituting for $\phi_{0}$ in (14) to (19) and transforming the various equations and conditions, we obtain that

$$
\frac{d^{2} \hat{\psi}_{0}}{d y^{2}}-\xi^{2} \hat{\psi}_{0}=A \xi e^{-\lambda y},
$$

where $A=i \lambda\left(1-R_{0}\right)-i k\left(1+R_{0}\right)$, with

$$
\frac{d \hat{\psi}_{0}}{d y}+\lambda \hat{\psi}_{0}=0 \quad \text { on } \quad y=0
$$

and

$$
\left|\nabla \hat{\psi}_{0}\right| \rightarrow \infty \text { as } y \rightarrow \infty .
$$

The solution of the differential equation (28) along with the boundary conditions (29) and (30) is given by

$$
\hat{\psi}_{0}=A \xi e^{-\lambda y} /\left(\lambda^{2}-\xi^{2}\right) \quad(\xi \neq \lambda) .
$$

We observe that the expression (31) for $\hat{\psi}_{0}$ involves an unknown constant $A$; in order to determine this constant, we use the idea that $\hat{\psi}_{0}$ is a Fourier transform which cannot have a singularity on the real $\xi$ axis. This forces the result that

$$
\lim _{\xi \rightarrow \lambda}(\xi-\lambda) \hat{\psi}_{0}(\xi, y)=0
$$

so that we obtain $A=0$, which implies that

$$
R_{0}=(\lambda-k) /(\lambda+k) .
$$

Again, considering the inverse transform of $\hat{\psi}_{0}$ and the condition on $\psi_{0}$ as $x \rightarrow \infty$, we obtain that

$$
\psi_{0}=0 \text {. }
$$

This gives the solution $\phi_{0}(x, y)$ in the form

$$
\phi_{0}(x, y)=\phi_{i}(x, y)+R_{0} \phi_{i}(-x, y),
$$

where $R_{0}$ is given by (33). 
3.2. Determination of $\phi_{1}(x, y)$ (Infinite depth case) Putting $\phi_{1}(x, y)=\phi_{1}(x, y)+$ $R_{1} \phi_{i}(-x, y)$ for the problem $P_{1}$ and using the transform defined in (27), we have

$$
\frac{d^{2} \hat{\psi}_{1}}{d y^{2}}-\xi^{2} \hat{\psi}_{1}=\xi\left(A e^{-\lambda y}+f(y)\right)
$$

where $A=-i(k+\lambda) R_{1}$, with the conditions

$$
\frac{d \hat{\psi}_{1}}{d y}+\lambda \hat{\psi}_{1}=0 \quad \text { on } y=0
$$

and

$$
\left|\nabla \hat{\psi}_{1}\right| \rightarrow 0 \quad \text { as } y \rightarrow \infty .
$$

It is straightforward to obtain the function $\hat{\psi}_{1}$ by using the Green's function technique (see Friedman [3]). We find that

$$
\hat{\psi}_{1}(\xi, y)=\int_{0}^{\infty}\left(\xi\left(A e^{-\lambda s}+f(s)\right)\right) G(y, s) d s,
$$

where the Green's function $G$ is defined by

$$
G(y, s)=\left\{\begin{array}{lll}
\frac{\xi \cosh \xi s-\lambda \sinh \xi s}{(\lambda-\xi) \xi} e^{-\xi y} & \text { for } \quad y \geq s \\
\frac{\xi \cosh \xi y-\lambda \sinh \xi y}{(\lambda-\xi) \xi} e^{-\xi s} & \text { for } \quad y \leq s .
\end{array}\right.
$$

The unknown constant $A$ in (39) can be determined in this case by using the regularity condition used in the case discussed above. We obtain that

$$
A=-2 \lambda \int_{0}^{\infty} e^{-\lambda s} f(s) d s
$$

which implies

$$
R_{1}=\frac{2 \lambda \int_{0}^{\infty} e^{-\lambda s} f(s) d s}{i(k+\lambda)}
$$

Next, inverting the transform $\hat{\psi}_{1}(\xi, y)$ given in (27), we derive that

$$
\psi_{1}(x, y)=c_{0}(y) e^{-i k x}+\frac{2}{\pi} \int_{0}^{\infty} \frac{\hat{\psi}_{1}(\xi, y)(\xi \cos \xi x-i k \sin \xi x)}{\xi^{2}-k^{2}} d \xi,
$$

in which (and in later occurrences) singular integrals are understood in the sense of Cauchy principal values.

In (42) the arbitrary function $c_{0}(y)$ is to be determined by using the condition that $\psi \rightarrow 0$ as $x \rightarrow \infty$. Now writing $(\xi \cos \xi x-i k \sin \xi x)=\frac{1}{2}\left((\xi-k) e^{i \xi k}+(\xi+k) e^{-i \xi k}\right)$ and rotating the contour along the positive imaginary axis for the integral involving 
$e^{i \xi x}$ and along the negative imaginary axis for the integral involving $e^{-i \xi x}$, we can write the expression for $\psi_{1}(x, y)$ in (42) in another form $\psi_{1}(x, y)=c_{0}(y) e^{-i k x}+i \hat{\psi}_{1}(k, y) e^{-i k x}+\int_{0}^{\infty} \exp (-\eta x) A_{0}(\eta)(\eta \cos \eta y-\lambda \sin \eta y) d \eta$.

where

$$
A_{0}(\eta)=-\frac{2}{\pi} \int_{0}^{\infty} \frac{f(s)(\eta \cos \eta s-\lambda \sin \lambda s)}{\left(i \eta+\frac{b \rho \omega}{\mu}\right)\left(\lambda^{2}+\eta^{2}\right)} d s .
$$

This form of $\psi_{1}$ along with the condition at infinity suggest that

$$
c_{0}(y)=-i \hat{\psi}_{1}(k, y)
$$

and then

$$
\psi_{1}(x, y)=\int_{0}^{\infty} \exp (-\eta x) A_{0}(\eta)(\eta \cos \eta y-\lambda \sin \eta y) d \eta,
$$

where $A_{0}(\eta)$ is given in (44). We get

$$
\phi_{1}(x, y)=\psi_{1}(x, y)+R_{1} \phi_{i}(-x, y),
$$

where $\psi_{1}(x, y)$ and $R_{1}$ are as given above. This completes the solution of the problem in this case.

3.3. Determination of $\phi_{0}(x, y)$ (Finite depth case) Proceeding in the same way as in the case of infinite depth, we find that

$$
\phi_{0}(x, y)=\phi_{i}(x, y)+R_{0} \phi_{i}(-x, y)
$$

for problem $P_{2}$, where

$$
R_{0}=\left(k_{0}-k\right) /\left(k_{0}+k\right) .
$$

3.4. Determination of $\phi_{1}(x, y)$ (Finite depth case) Putting $\phi_{1}=\psi_{1}+R_{1} \phi_{i}(-x, y)$ for problem $P_{2}$ and using the transform as defined in (27), we obtain the ordinary differential equation

$$
\frac{d^{2}}{d y^{2}} \hat{\psi}_{1}-\xi^{2} \hat{\psi}_{1}=-i \xi\left(R_{1}\left(k+k_{0}\right) \frac{\cosh k_{0}(h-y)}{\cosh k_{0} h}+f(y)\right) \quad 0<y<h,
$$

with the conditions that

$$
\frac{d}{d y} \hat{\psi}_{1}+\lambda \hat{\psi}_{1}=0 \quad \text { on } \quad y=0
$$


and

$$
\frac{d \hat{\psi}_{1}}{d y}=0 \text { on } y=h .
$$

Using the Green's function technique as before, we obtain the solution of the problem posed by the relations (50) to (52) in the form

$$
\hat{\psi}_{1}(\xi, y)=-i \xi \int_{0}^{h} G(y, s)\left[R_{1}\left(k+k_{0}\right) \frac{\cosh k_{0}(h-s)}{\cosh k_{0} h}+f(s)\right] d s,
$$

where $G(y, s)$ is given by

$$
G(y, s)=\left\{\begin{array}{lll}
\frac{\xi \cosh \xi s-\lambda \sinh \xi s}{(\lambda \cosh \xi h-\xi \sinh \xi h) \xi} \cosh \xi(h-y) & \text { for } \quad y \geq s \\
\frac{\xi \cosh \xi y-\lambda \sinh \xi y}{(\lambda \cosh \xi h-\xi \sinh \xi h) \xi} \cosh \xi(h-s) & \text { for } \quad y \leq s
\end{array}\right.
$$

Now the expression $\left(\lambda \cosh \xi h-\xi \sinh \xi h\right.$ ) has a zero at $\xi=k_{0}$ on the real axis, so that $\hat{\psi}_{1}(\xi, y)$ has an apparent singularity on the real axis at $\xi=k_{0}$. Using the regularity of the transform as before, we obtain

$$
R_{1}=\frac{-4 i k_{0} \cosh k_{0} h \int_{0}^{h} f(s) \cosh k_{0}(h-s) d s}{\left(k+k_{0}\right)\left(\sinh 2 k_{0} h+2 k_{0} h\right)} .
$$

Finally, taking the inverse transform of $\hat{\psi}_{1}(\xi, y)$, we derive that

$$
\psi_{1}(x, y)=c_{1}(y) e^{-i k x}+\frac{2}{\pi} \int_{0}^{\infty} \frac{\hat{\psi}_{1}(\xi, y)(\xi \cos \xi x-i k \sin \xi x)}{\xi^{2}-k^{2}} d \xi,
$$

where $c_{1}(y)$ is to be determined by using the condition at infinity on $\psi_{1}(x, y)$. Transforming the integral in (56) into one over the imaginary axis as was done in the case of infinite depth and using the fact that $\xi= \pm i k_{n}(n=1,2, \ldots)$ are the zeros of $(\lambda \cosh \xi h-\xi \sinh \xi h)=0$, we obtain

$$
c_{1}(y)=-i \hat{\psi}_{1}(k, y)
$$

and $\psi_{1}(x, y)$ is given by

$$
\psi_{1}(x, y)=\sum_{n=1}^{\infty} B_{n} e^{-k_{n} x} \cos k_{n}(h-y),
$$

where

$$
B_{n}=-\frac{4\left(k_{n}+i k\right) k_{n} \int_{0}^{h} f(s) \cos k_{n}(h-s) d s}{\left(k_{n}^{2}-k^{2}\right)\left(2 k_{n} h+\sin 2 k_{n} h\right)} .
$$

Finally, we obtain the solution of the problem for the case of water of finite depth as

$$
\phi_{1}(x, y)=R_{1} \phi_{i}(-x, y)+\psi_{1}(x, y),
$$

where $R_{1}$ is given in (54) and $\psi_{1}$ by (57). 


\section{Particular shape of the wall}

As an illustration, we have calculated the reflection coefficient and the value of $f(y)$ for a particular shape of the cliff, for which $c(y)=y e^{-p y}$ for $p>0$. Then $f(y)$ and $R_{1}$ are given by

$$
\begin{gathered}
f(y)=\frac{2 \lambda e^{-(p+\lambda) y}}{\left(\lambda+\frac{b \rho \omega}{\mu}\right)}\left[\lambda((p+\lambda) y-1)-\left(\frac{b \rho \omega}{\mu}\right)^{2} y\right], \\
R_{1}=\frac{i 4 \lambda^{2}\left(\lambda^{2}+\left(\frac{b \rho \omega}{\mu}\right)^{2}\right)}{\left(\lambda+\frac{b \rho \omega}{\mu}\right)^{2}(p+2 \lambda)^{2}},
\end{gathered}
$$

for water of infinite depth, and

$$
\begin{aligned}
& f(y)=-\frac{2 k_{0} e^{-p y}\left[k_{0}\left(\sinh k_{0}(h-y)-p y \sinh k_{0}(h-y)-y k_{0} \cosh k_{0}(h-y)\right)+B\right]}{\left(k_{0}+\frac{b \rho \omega}{\mu}\right) \cosh k_{0} h}, \\
& R_{1}=\frac{8 i k_{0}^{2}\left[\left(k_{0}^{2}+\left(\frac{b \rho \omega}{\mu}\right)^{2}\right)\left(\frac{\left(p^{2}+4 k_{0}^{2}\right) \cosh 2 k_{0} h-4 p k_{0} \sinh 2 k_{0} h}{2\left(p^{2}-4 k_{0}^{2}\right)^{2}}+\frac{1-e^{-p h}(1+p h)}{2 p^{2}}\right)+C\right]}{E} \text { for } p \neq 2 k_{0}
\end{aligned}
$$

and

$$
R_{1}=4 i p^{2}\left(\frac{\frac{e^{p h}}{64}-\frac{1}{8}-\frac{p e^{-p h}}{2}\left(\frac{p h^{2}}{8}+\frac{3 h}{8}+\frac{7}{16 p}\right)+\left(\frac{b \rho \omega}{\mu}\right)^{2}\left[\frac{e^{p h}}{16 p^{2}}+\frac{1}{2 p^{2}}+D\right]}{(\sinh p h+p h)\left(k_{0}+\frac{b \rho \omega}{\mu}\right)^{2}}\right) \text { for } p=2 k_{0} \text {, }
$$

where $B=\left(\frac{b \rho \omega}{\mu}\right)^{2} \cosh k_{0}(h-y), C=\frac{(p h+1) k_{0}^{2}+(p h-1)\left(\frac{b \rho \omega}{\mu}\right)^{2}}{2\left(p^{2}-4 k_{0}^{2}\right)}, D=\frac{e^{-p h}}{2}\left(\frac{h^{2}}{4}-\frac{5 h}{4 p}-\frac{9}{8 p^{2}}\right)$, and $E=\left(k_{0}+\frac{b \rho \omega}{\mu}\right)^{2}\left(\sinh 2 k_{0} h+2 k_{0} h\right)$ for water of finite depth. Here one can see that as $h \rightarrow \infty, R_{1}$ is the same in both cases.

\section{Numerical results and discussion}

The magnitude of $\left|R_{1}\right|$ has been calculated for both water of finite and infinite depth, for different cases, and the numerical results have been presented graphically. It is observed that $\left|R_{1}\right|$ varies between zero and one in both cases, as is to be expected.

In case of water of finite depth, $\left|R_{1}\right|$ is a function of $p,(b \rho \omega) / \mu$ and $\lambda$. Results varying one variable and keeping the other two variables fixed have been presented in Figure 3. From the figure it is clear that as porosity $(b \rho \omega) / \mu$ increases, $\left|R_{1}\right|$ 
initially decreases and then goes on increasing; $p$ increases as $\left|R_{1}\right|$ decreases, and as $\lambda$ increases, $\left|R_{1}\right|$ increases.

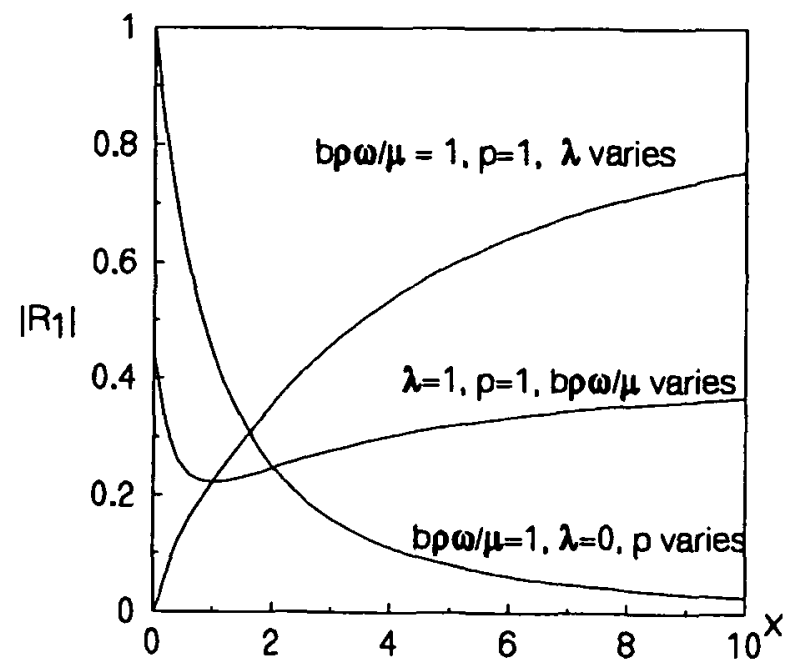

FIGURE 3. $\left|R_{l}\right|$ for water of infinite depth. $\lambda, p, b \rho \omega / \mu$ vary along $x$-axis.

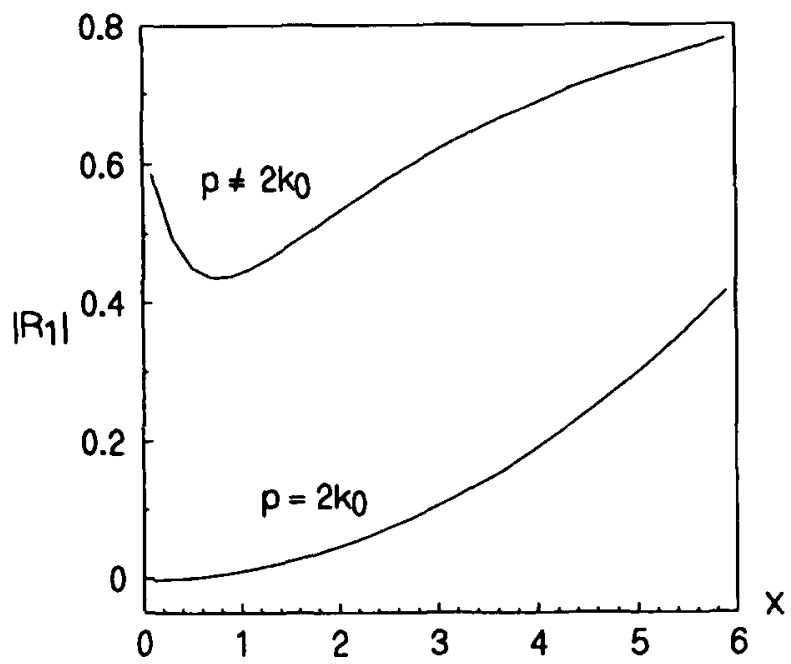

FIGURE 4. $\left|R_{1}\right|$ for water of finite depth. $\lambda=1, h=1, b \rho \omega / \mu$ varies along $x$-axis.

In the case of water of finite depth, one can see that as $h$ increases, $\lambda$ becomes equal to $k_{0}$. Hence the results for the case of infinite depth hold good for large $h$. Here there are two cases. In the case $p \neq 2 k_{0}$, the value of $p$ has been considered as 


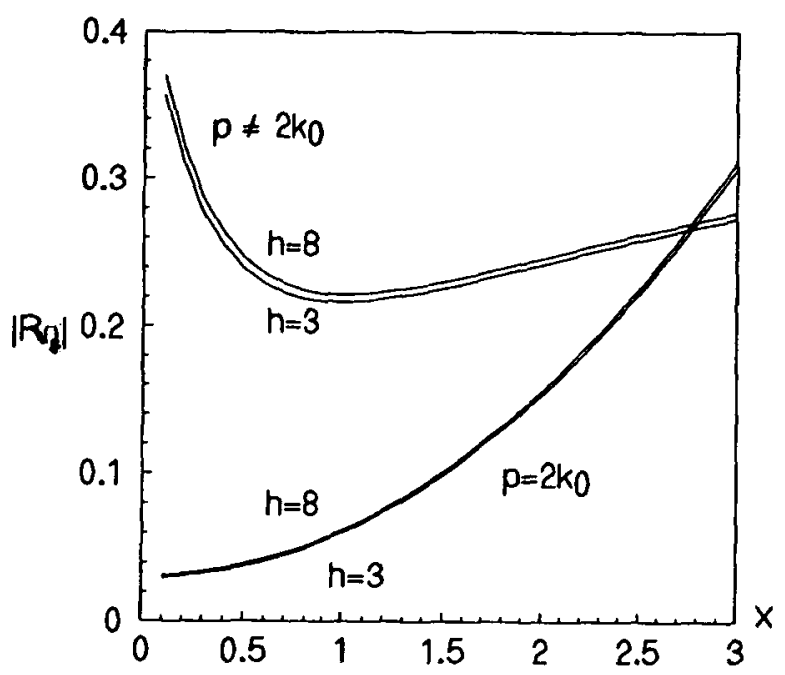

FIGURE 5. $\left|R_{1}\right|$ for water of finite depth. $p=1, \lambda=1, b p \omega / \mu$ varies along $x$-axis.

$p=1$. In Figures 4 and 5 for different values of $h,\left|R_{1}\right|$ has been plotted separately along with $(b \rho \omega) / \mu$. It is observed that for $h=5$ onwards the values of $\left|R_{1}\right|$ become numerically equal and the graphs overlap. From Figure 6 it is observed that increases in $h$ initially increase the value of $\left|R_{1}\right|$ in the case $p=2 k_{0}$ and decrease the value of $\left|R_{1}\right|$ in the case $p \neq 2 k_{0}$; after a certain stage, $\left|R_{1}\right|$ remains constant in both cases.

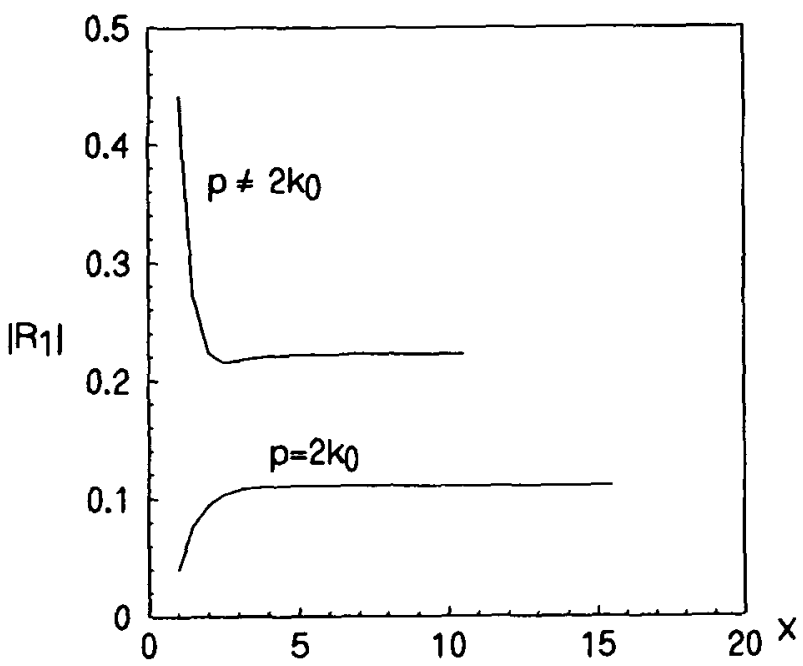

FIGURE 6. $\left|R_{1}\right|$ for water of finite depth. $\lambda=1, b \rho \omega / \mu=1, h$ varies along $x$-axis. 


\section{Conclusion}

A more general problem of water wave scattering involving a nearly vertical porous wall has been handled completely, and as a particular case of this problem, the results available for much simpler problems have been rederived.

\section{Acknowledgements}

We are grateful to the referees for their valuable suggestions improving the presentation of the paper a great deal.

\section{References}

[1] A. Chakrabarti, "A note on the porous-wavemaker problem", Acta Mechanica 77 (1989) 121-129.

[2] A. T. Chwang, "A porous wavemaker theory", J. Fluid Mechanics 132 (1983) 395-406.

[3] B. Friedman, Principles and techniques of applied mathematics (John Wiley \& Sons, 1956).

[4] P. J. Kachoyan and W. D. McKee, "Wave forces on steeply sloping sea walls", J. Eng. Math. 19 (1985) 351-362.

[5] B. N. Mandal and A. Chakrabarti, "A note on diffraction of water waves by a nearly vertical barrier", IMA J. Appl. Math. 43 (1989) 157-165.

[6] B. N. Mandal and S. K. Kar, "Reflection of water waves by a nearly vertical wall", Int'l. J. Math. Educ. Sci. and Technology 23 (5) (1992) 665-670.

[7] D. C. Shaw, "Perturbational results for diffraction of water waves by nearly vertical barriers", IMA J. Appl. Math. 33 (1985) 99-117.

[8] I. N. Sneddon, The use of integral transforms (Tata McGraw Hill, New Delhi, 1974) 70-75. 